\title{
molecules
}

ISSN 1420-3049

www.mdpi.com/journal/molecules

Communication

\section{New Flavonolignan Glycosides from the Aerial Parts of Zizania latifolia}

\section{Seung-Su Lee ${ }^{1,2}$, Nam-In Baek ${ }^{1,3}$, Yoon-Su Baek ${ }^{3}$, Dae-Kyun Chung ${ }^{1,2}$, Myoung-Chong Song ${ }^{4}$ and Myun-Ho Bang 2,3,*}

1 Graduate School of Biotechnology and Research Institutee of life Science \& Resources, Kyung Hee University, Yongin-si, Gyeonggi-do 446-701, Korea;

E-Mails: 1dkhhghh@khu.ac.kr (S.-S.L.); nibaek@khu.ac.kr (N.-I.B.); dkchung@khu.ac.kr (D.-K.C.)

2 Skin Biotechnology Center, Kyung Hee University, Suwon 433-766, Korea

3 Department of Oriental Medicinal Material and Processing, College of Life Science, Kyung Hee University, Yongin-si, Gyeonggi-do 446-701, Korea; E-Mail: acplant@khu.ac.kr

4 Department of Chemistry and Nano Science, Ewha Womans University, Seoul 120-750, Korea; E-Mail: smch517@hanmail.net

* Author to whom correspondence should be addressed; E-Mail: bangmh68@khu.ac.kr; Tel.: +82-31-888-6175; Fax: +82-31-888-6173.

Academic Editor: Derek J. McPhee

Received: 6 March 2015 / Accepted: 24 March 2015 / Published: 30 March 2015

\begin{abstract}
Two new flavonolignan glycosides, tricin-4'-O-(threo- $\beta$-guaiacylglyceryl) ether 7"-O- $\beta$-D-glucopyranose (4) and tricin-4'-O-(erythro- $\beta$-guaiacylglyceryl) ether 7 "-O- $\beta$-D-glucopyranose (5) were isolated from the roots of Zizania latifolia, together with tricin-7-O- $\beta$-D-glucopyranose (1), tricin-4'-O-(threo- $\beta$-guaiacylglyceryl) ether 7-O- $\beta$-D-glucopyranose (2), and tricin-4'-O-(erythro- $\beta$-guaiacylglyceryl) ether 7-O- $\beta$-D-glucopyranose (3). Their structures were identified on the basis of spectroscopic techniques, including HR-ESI/MS, 1D-NMR $\left({ }^{1} \mathrm{H},{ }^{13} \mathrm{C}, \mathrm{DEPT}\right), 2 \mathrm{D}-\mathrm{NMR}$ (gCOSY, gHSQC, gHMBC), and IR spectroscopy.
\end{abstract}

Keywords: Zizania latifolia; flavonolignan glycoside; tricin-7-O- $\beta$-D-glucopyranose 


\section{Introduction}

Zizania latifolia Turcz (wild rice) is cultivated in various regions of Southeastern Asia, including Korea, Japan, and China. Particularly, in China, wild rice grain is used as an important traditional medicine to treat anemia and fever in addition to heart, kidney, and liver disorders. It has been reported that wild rice species possess rich nutrient content [1,2], as well as diverse biological activities such as anti-obesity [3], anti-oxidant [4], anti-inflammatory and anti-allergenic effects [5]. Previous phytochemical studies on Z. latifolia have confirmed the presence of flavonolignan groups along with tricin. In order to identify phytochemical components of $Z$. latifolia, isolation and structural determination of two new flavonolignan glycosides, tricin-4'-O-(threo- $\beta$-guaiacylglyceryl) ether 7"-O- $\beta$-D-glucopyranose (4) and tricin-4'-O-(erythro- $\beta$-guaiacylglyceryl) ether 7"-O- $\beta$-D-glucopyranose (5), a flavone glycoside, tricin-7-O- $\beta$-D-glucopyranose (1), two known flavonolignan glycosides tricin-4'-O-(threo- $\beta$ guaiacylglyceryl) ether 7-O- $\beta$-D-glucopyranose (2), and tricin-4'-O-(erythro- $\beta$-guaiacylglyceryl) ether 7-O- $\beta$-D-glucopyranose (3) were performed. These compounds are of particular interest since flavonolignan glycosides have been reported for the first time from Z. latifolia (Figure 1).

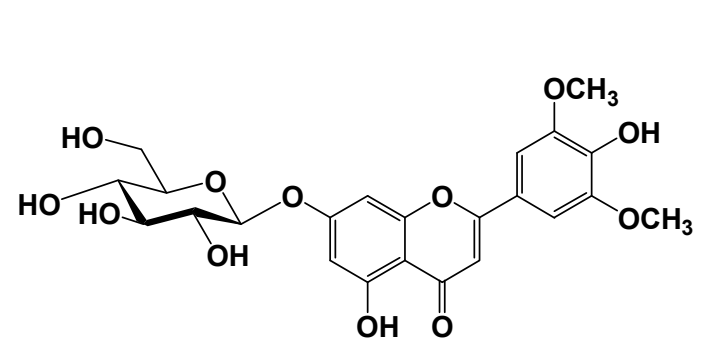

Compound 1

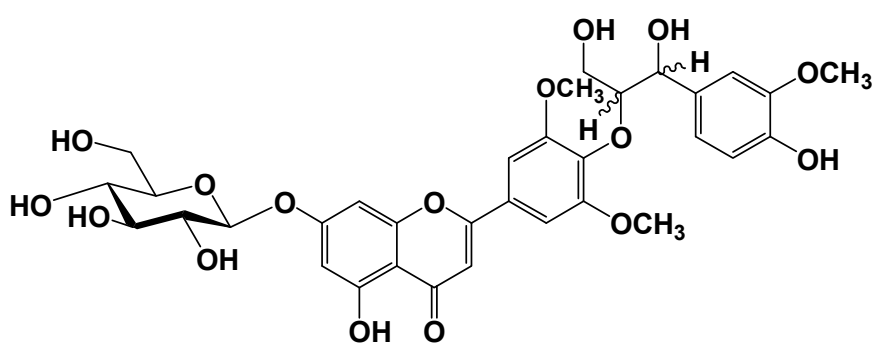

Compound 2: threo

Compound 3: erythro

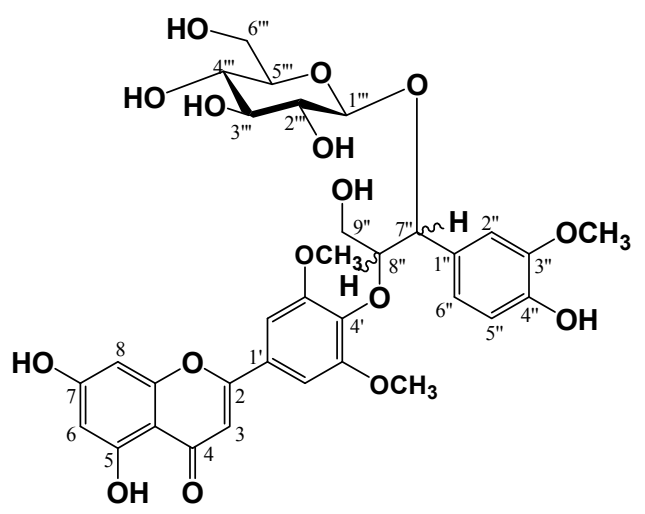

Compound 4: threo

Compound 5: erythro

Figure 1. Chemical structures of isolated compounds 1-5.

\section{Results and Discussion}

The aerial parts were extracted using $80 \% \mathrm{MeOH}$, and the concentrated extracts were successively partitioned using EtOAc, n-BuOH, and $\mathrm{H}_{2} \mathrm{O}$. Repeated $\mathrm{SiO}_{2}$, and ODS-A column chromatographic separation of the EtOAc fraction produced a purified flavone glycoside, and four flavonolignans 
including two new flavonolignan glycosides. These compounds were identified as tricin-7- $O-\beta$-Dglucopyranose (1), tricin-4'-O-(threo- $\beta$-guaiacylglyceryl) ether 7-O- $\beta$-D-glucopyranose (2), tricin-4'- $O$ (erythro- $\beta$-guaiacylglyceryl) ether 7-O- $\beta$-D-glucopyranose (3), tricin-4'-O-(threo- $\beta$-guaiacylglyceryl) ether 7"-O- $\beta$-D-glucopyranose (4) and tricin-4'-O-(erythro- $\beta$-guaiacylglyceryl) ether 7"-O- $\beta$-D-glucopyranose (5), respectively, based on interpretation of the spectroscopic data including NMR, MS, and IR spectrascopy, and confirmed by comparison of the data with those reported in the literature [6-8]. All compounds were isolated for the first time from Z. latifolia.

Compound 4 (a yellow amorphous powder) was developed by TLC, followed by spraying with $10 \%$ $\mathrm{H}_{2} \mathrm{SO}_{4}$ and heating to produce a dark yellow color. In the ${ }^{1} \mathrm{H}-\mathrm{NMR}$ spectrum, three olefin methine proton signals at $\delta 7.50(1 \mathrm{H}, \mathrm{d}, J=1.6 \mathrm{~Hz}, \mathrm{H}-2 "), 7.37(1 \mathrm{H}, \mathrm{dd}, J=8.0,1.6 \mathrm{~Hz}, \mathrm{H}-6 ")$, and $7.18(1 \mathrm{H}, \mathrm{d}$, $J=8.0 \mathrm{~Hz}, \mathrm{H}-5 ")$ were observed due to a 1,2,4-trisubstituted benzene ring along with five olefine methine proton signals at $\delta 7.27(2 \mathrm{H}$, br. s, H-2', 6'), $6.97(1 \mathrm{H}, \mathrm{s}, \mathrm{H}-3), 6.83(1 \mathrm{H}, \mathrm{d}, J=2.0 \mathrm{~Hz}, \mathrm{H}-8)$, and $6.72(1 \mathrm{H}, \mathrm{d}, J=2.0 \mathrm{~Hz}, \mathrm{H}-6)$ due to a flavone moiety. In the oxygenated region, two oxygenated methylene proton signals at $\delta 5.89(1 \mathrm{H}, \mathrm{d}, J=6.0 \mathrm{~Hz}, \mathrm{H}-7 ")$ and $5.19\left(1 \mathrm{H}, \mathrm{m}, \mathrm{H}-8^{\prime \prime}\right)$, an oxygenated methylene proton signal at $\delta 4.75(1 \mathrm{H}, \mathrm{dd}, J=12.4,3.6 \mathrm{~Hz}, \mathrm{H}-9$ "a) and $4.36(1 \mathrm{H}, \mathrm{dd}, J=12.0,2.8 \mathrm{~Hz}$,

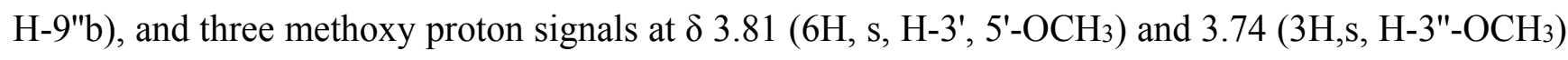
were observed. There were also oxygenated methine and methylene signals for a monosaccharide moiety observed in the region from $\delta 4.12$ to 4.28 , including one hemiacetal proton signal at $\delta 5.54$

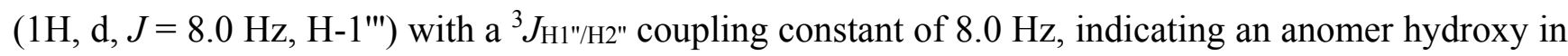
$\beta$-configuration. The relative configuration of chiral carbons, C-7" and C-8", in compound (4) was deduced to be threo type from the coupling constant $(J=6.0 \mathrm{~Hz})$ between the oxygenated methine signals $\mathrm{H}-7$ " ( $\left.\delta_{\mathrm{H}} 5.89\right)$ and $\mathrm{H}-8$ " ( $\left.\delta_{\mathrm{H}} 5.19\right)$. It was reported that the $\mathrm{J}_{\mathrm{H}-7 "}, 8$ " coupling constant of proton resonance in the guaiacylglyceryl moiety can be used to distinguish between the erythro and threo forms, with $J$ values of 4.0-5.0 and 6.0-7.0 Hz, respectively [9-11]. The ${ }^{13} \mathrm{C}-\mathrm{NMR}$ spectrum showed 33 carbon signals including three methoxy carbons $\left[\delta 52.3\left(\mathrm{C}-3^{\prime}, 5^{\prime}-\mathrm{OCH}_{3}\right)\right.$ and $56.6(\mathrm{C}-3$ '--OCH$\left.)\right]$, confirming that compound $\mathbf{4}$ was a flavonoid with a phenylpropanoid moiety and hexose moiety. In the low-magnetic field region, a conjugated ketone carbon $\delta 183.9$ (C-4), seven oxygenated olefine quaternary carbons $[\delta 167.3$ (C-7), 165.0 (C-2), 164.3 (C-5), 159.7 (C-9), 155.1 (C-3', 5'), 141.3 $\left(\mathrm{C}-4{ }^{\prime}\right)$ ], two olefine quaternary carbons $\left[\delta 127.9\left(\mathrm{C}-1^{\prime}\right), 106.3(\mathrm{C}-10)\right]$, and five olefin methine carbons [ $\delta 107.0$ (C-3), 105.9 (C-2', 6'), 101.3 (C-6), 96.3 (C-8)] signals were observed, indicating the presence of a flavone moiety. In addition, two oxygenated olefine quaternary carbons [ $\delta 149.8(\mathrm{C}-3 "), 149.1$ $\left(\mathrm{C}-4^{\prime \prime}\right)$, one olefine quaternary carbon $\delta 133.1$ (C-1"), and three olefine methine carbons $[\delta 122.8$ (C-6"), 117.0 (C-5"), 114.0 (C-2")], two oxygenated methine carbons [ 87.8 (C-8"), 82.6 (C-7")] and one oxygenated methylene carbon $\delta 62.7\left(\mathrm{C}-9^{\prime \prime}\right)$ signals were observed, indicating the presence of a phenylpropanoid moiety. In the HMBC spectrum, the oxygenated methine proton $\delta \mathrm{H} 5.19(1 \mathrm{H}, \mathrm{m}$, $\left.\mathrm{H}-8^{\prime \prime}\right)$ showed a correlated cross peak with an oxygenated olefine quaternary carbon $\delta_{\mathrm{C}} 141.3\left(\mathrm{C}-4^{\prime}\right)$, indicating that the flavone and phenylpropanoid moieties were linked between $\delta_{\mathrm{C}} \mathrm{C}-8^{\prime \prime}$ and $\delta \mathrm{C} C-4$ ' via an ether linkage, as well as a cross-peak between an anomer proton signal at $\delta_{\mathrm{H}} 5.54(1 \mathrm{H}$, d, $\left.J=8.0 \mathrm{~Hz}, \mathrm{H}-1{ }^{\prime \prime \prime}\right)$ and an oxygenated methine carbon signal at $\delta \mathrm{C} 82.6$ (C-7"), indicating that glucose was attached to the $\mathrm{C}-7$ " of the phenylpropanoid through a glycosidic likage (Figure 2). Finally, the structure of compound (4) was determined to be tricin-4'-O-(threo- $\beta$-guaiacylglyceryl) ether 7"-O- $\beta$-Dgluco-pyranose, which is salcolin A 7"-O- $\beta$-D-glucopyranose. The molecular weight was determined to be 
688.2003 from the pseudomolecular ion peak at $\mathrm{m} / z 687.1962[\mathrm{M}-\mathrm{H}]^{-}$in the negative high resolution ESI-MS (calculated for $\mathrm{C}_{33} \mathrm{H}_{35} \mathrm{O}_{16}$ ).

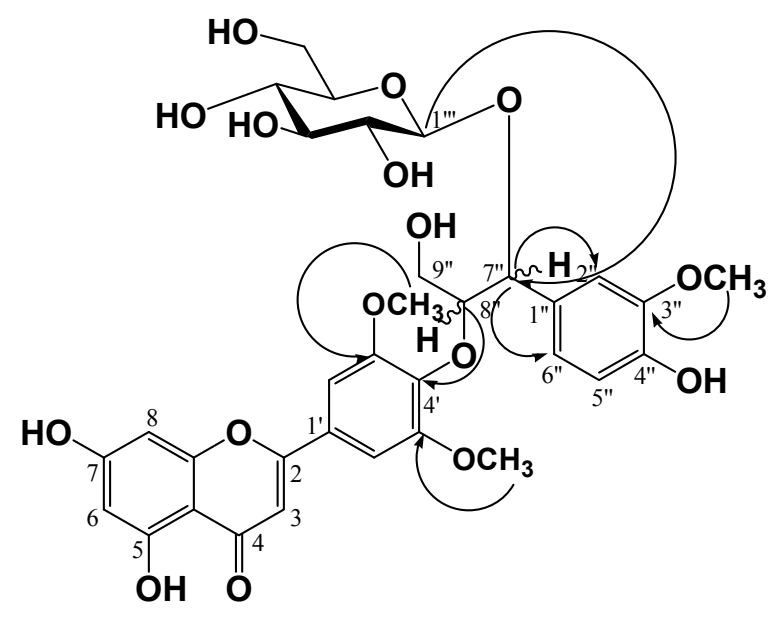

Figure 2. Key HMBC correlations of compound 4.

Signals in the ${ }^{1} \mathrm{H}-\mathrm{NMR}\left(400 \mathrm{MHz}\right.$, pyridine- $\left.d_{5}, \delta_{\mathrm{H}}\right)$ and ${ }^{13} \mathrm{C}-\mathrm{NMR}\left(100 \mathrm{MHz}\right.$, pyridine- $\left.d_{5}, \delta_{\mathrm{C}}\right)$ spectra of compound 5 were almost the same as those of compound 4 , with the exception of the relative stereostructure of C-7" and C-8". Compound 5 was confirmed to be erythro type from the coupling constant between the oxygenated methine signals of $\mathrm{H}-7$ " and $\mathrm{H}-8$ " $(J=4.8 \mathrm{~Hz})$. The ether linkage (C-8"/C-4') and glycosidic likage (C-1"'/C-7") were confirmed from the cross peak between the oxygenated methine proton $\delta_{\mathrm{H}} 5.16\left(1 \mathrm{H}, \mathrm{m}, \mathrm{H}-8^{\prime \prime}\right)$ and oxygenated olefine quaternary carbon $\delta_{\mathrm{C}} 142.0$ (C-4'), as well as the cross-peak between the anomer proton signal at $\delta_{\mathrm{H}} 5.35\left(1 \mathrm{H}, \mathrm{d}, J=8.0 \mathrm{~Hz}, \mathrm{H}-1^{\prime \prime \prime}\right)$ and a oxygenated methine carbon signal at $\delta_{\mathrm{C}} 81.9$ (C-7") in the HMBC spectrum. Consequently, the structure of compound 5 was determined to be tricin-4'-O-(erythro- $\beta$-guaiacylglyceryl) ether 7"-O- $\beta$-Dglucopyranose, which is salcolin B 7"-O- $\beta$-D-glucopyranose. The molecular weight was determined to be 688.2003 from the pseudomolecular ion peak at $m / z 687.1952[\mathrm{M}-\mathrm{H}]^{-}$in the negative high resolution ESI-MS (calculated for $\mathrm{C}_{33} \mathrm{H}_{35} \mathrm{O}_{16}$ ).

\section{Experimental}

\subsection{General}

Preparative-liquid chromatography (prep-LC) (LC-Forte/R, YMC, Kyoto, Japan) was carried out using cartridge YMC-DispoPack (ODS-25, 80 g, and 120 g, YMC). Silica gel (230-400 mesh, Merck, Darmastdt, Germany) and Sephadex LH-20 (Merck) were used for column chromatography. Thin-layer Chromatography (TLC) analysis was performed using on a Kiesel gel 60F 254 plate (Merck) and a RP-18 F254s plates (Merck), and detection was performed by a UV lamp at 254 and $365 \mathrm{~nm}$ and $10 \% \mathrm{H}_{2} \mathrm{SO}_{4}$ solution by spraying and heating. ${ }^{1} \mathrm{H}$ and ${ }^{13} \mathrm{C}-\mathrm{NMR}$ along with $2 \mathrm{D}-\mathrm{NMR}$ data were obtained on a Bruker AVANCE II $400\left({ }^{1} \mathrm{H}-\mathrm{NMR}\right.$ at $400 \mathrm{MHz},{ }^{13} \mathrm{C}-\mathrm{NMR}$ at $\left.100 \mathrm{MHz}\right)$ spectrometer (Bruker, Rheinstetten, Germany) in pyridine- $d 5$ with TMS as internal standard. ESI/MS was obtained on a AB SCIEX triple TOF 5600 mass spectrometer (AB SCIEX, Concord, ON, Canada). All the reagent grade chemicals were purchased from Sigma Chemical Co. (St. Louis, MO, USA). 


\subsection{Plant Material}

Plant sample. Dried aerial parts of Z. latifolia was purchased from store located at Yeongcheon City, Gyeongbuk, Korea. A voucher specimen (KHU-0130301) was deposited at the Laboratory of Natural Products Chemistry, Kyung Hee University, Yongin, Korea.

\subsection{Extraction and Isolation}

Dried powder $(5 \mathrm{~kg}$ ) was extracted at room temperature with $80 \% \mathrm{MeOH}(45 \mathrm{~L})$. The concentrated extract was partitioned with water $(3 \mathrm{~L})$, EtOAc $(3 \mathrm{~L})$ and $\mathrm{n}-\mathrm{BuOH}(3 \mathrm{~L})$ successively to produce. EtOAc extract ( $97 \mathrm{~g}, \mathrm{ZLE}), \mathrm{n}-\mathrm{BuOH}$ extract (240 g, ZLB) and $\mathrm{H}_{2} \mathrm{O}$ extract (57 g, ZLW). The EtOAc extract was applied to a silica gel c.c. $(12 \times 14 \mathrm{~cm})$, and eluted with $\mathrm{CHCl}_{3}: \mathrm{MeOH}(50: 1 \rightarrow 30: 1 \rightarrow$ $20: 1 \rightarrow 12: 1 \rightarrow 10: 1 \rightarrow 7: 1 \rightarrow 5: 1 \rightarrow 3: 1 \rightarrow 1: 1$, each 3 L) monitoring by thin layer chromatography (TLC) to produce 13 fractions (ZLE-1 to ZLE-13). Fraction ZLE-12 (5.0 g) was combined and subjected to MPLC column chromatography: YMC-DispoPack AT (ODS-25:120 g, particle size: $25 \mu \mathrm{m})$. The fractions were analyzed by a UV detector at 254 and $356 \mathrm{~nm}$, and the mobile phase consisted of A: $0.1 \%$ formic acid in water, B: $0.1 \%$ formic acid in acetonitrile with a flow rate of $10 \mathrm{~mL} / \mathrm{min}$. The gradient consisted of $10 \% \mathrm{~B}$ held for $20 \mathrm{~min}$ to $30 \% \mathrm{~B}$ at a rate of $0.25 \% / \mathrm{min}$, from $30 \% \mathrm{~B}$ to $50 \% \mathrm{~B}$ at a rate of $0.20 \% / \mathrm{min}$, from $50 \% \mathrm{~B}$ to $70 \% \mathrm{~B}$ at a rate of $0.25 \% / \mathrm{min}$, and from $70 \%$ $\mathrm{B}$ to $90 \% \mathrm{~B}$ at a rate of $0.30 \% / \mathrm{min}$. A total 11 fractions (ZLE-12-1 to ZLE-12-15) were obtained. ZLE-12-10 (642 mg) was subjected to MPLC column chromatography: YMC-DispoPack AT (ODS-25: $80 \mathrm{~g}$, particle size: $25 \mu \mathrm{m}$ ), with A: $0.1 \%$ formic acid in water, B: $0.1 \%$ formic acid in acetonitrile as the mobile phase at a flow rate of $5 \mathrm{~mL} / \mathrm{min}$. The detector was maintained at 254 and $356 \mathrm{~nm}$. The gradient consisted of $20 \% \mathrm{~B}$ to $50 \% \mathrm{~B}$ at a rate of $0.5 \% / \mathrm{min}$, and $50 \% \mathrm{~B}$ to $70 \% \mathrm{~B}$ at a rate of $0.6 \% / \mathrm{min}$. Compound 1 ( $32 \mathrm{mg}, \mathrm{RT}=6.05 \mathrm{~min})$, compound 2 ( $35 \mathrm{mg}$, RT $=7.8 \mathrm{~min})$, compound 3 (42 mg, RT = $9.2 \mathrm{~min})$, compound 4 ( $22 \mathrm{mg}, \mathrm{RT}=12.4 \mathrm{~min})$ and compound $\mathbf{5}(31 \mathrm{mg}, \mathrm{RT}=14.6 \mathrm{~min})$ were obtained.

\subsection{Spectroscopic Data}

Tricin-7-O- $\beta$-D-glucopyranose (1). Pale yellow powder $\left(\mathrm{CH}_{3} \mathrm{OH}\right)$; negative ESI/MS m/z $491[\mathrm{M}-\mathrm{H}]^{-}$; IR (KBr) 3380, 2921, 1730, 1652, 1613, 1491,1358, $1084 \mathrm{~cm}^{-1} ;{ }^{1} \mathrm{H}-\mathrm{NMR}$ (pyridine-d5) $\delta 7.55$ (2H, s, H-2', 6'), $7.17(1 \mathrm{H}, \mathrm{d}, J=2.0 \mathrm{~Hz}, \mathrm{H}-8), 7.02(1 \mathrm{H}, \mathrm{s}, \mathrm{H}-3), 6.86(1 \mathrm{H}, \mathrm{d}, J=2.0 \mathrm{~Hz}, \mathrm{H}-6), 5.78$ (1H, d, $J=7.2 \mathrm{~Hz}, \mathrm{H}-1 "), 4.55$ (1H, dd, $J=10.4,1.2 \mathrm{~Hz}, \mathrm{H}-6$ "'a), 4.38-4.10 (5H, m, H-2"', H-3"', H-4"', H-5"', 6"'b-OCH $), 3.87$ (6H, s, OCH3-3', 5'). ${ }^{13} \mathrm{C}-\mathrm{NMR}$ (pyridine-d5) $\delta 182.8$ (C-4), 164.9 (C-2), 164.0 (C-7), 162.5 (C-5), 157.8 (C-9), 149.5 (C-3', 5'), 142.2 (C-4'), 121.0 (C-1'), 106.5 (C-10), 105.1 (C-2', 6'), 104.5 (C-3), 101.7 (C-1"), 100.6 (C-6), 95.5 (C-8), 79.2 (C-3"), 78.4 (C-5"), 74.7 (C-2"), 71.0 (C-4"), $62.2(\mathrm{C}-6 "), 56.5(\mathrm{C}-3$ ', 5'-OCH 3$)$.

Tricin-4'-O-(threo- $\beta$-guaiacylglyceryl) ether 7-O- $\beta$-D-glucopyranose (2). Yellow amorphous powder $(\mathrm{CH} 3 \mathrm{OH})$; Negative ESI/MS m/z $687[\mathrm{M}-\mathrm{H}]^{-}$; IR (KBr) 3369, 2938, 1652, 1611, 1588, 1512, 1495, 1356, 1263, 1159, 1124, $841 \mathrm{~cm}^{-1} ;{ }^{1} \mathrm{H}-\mathrm{NMR}$ (pyridine-d5) $\delta 7.53(1 \mathrm{H}, \mathrm{s}, \mathrm{H}-2 "), 7.37(1 \mathrm{H}, \mathrm{dd}, J=8.0$, $1.6 \mathrm{~Hz}, \mathrm{H}-6 "), 7.31$ (2H, s, H-2', 6'), 7.26 (1H, d, $J=8.0 \mathrm{~Hz}, \mathrm{H}-5 "), 7.14$ (1H, d, $J=2.4 \mathrm{~Hz}$, 
H-8), $7.01(1 \mathrm{H}, \mathrm{s}, \mathrm{H}-3), 6.85(1 \mathrm{H}, \mathrm{d}, J=2.0 \mathrm{~Hz}, \mathrm{H}-6), 5.76(1 \mathrm{H}, \mathrm{d}, J=7.2 \mathrm{~Hz}, \mathrm{H}-1 " '), 5.70$ (1H, d, $J=5.2 \mathrm{~Hz}, \mathrm{H}-7 "), 5.08$ (1H, q, $J=5.2,3.2 \mathrm{~Hz}, \mathrm{H}-8 "), 4.65$ (1H, dd, $J=12.0,5.2 \mathrm{~Hz}, \mathrm{H}-9 " \mathrm{e}), 4.54(1 \mathrm{H}$, dd, $J=12.0,2.4$ Hz, H-6"'a), 4.28 (1H, m, H-9"a), 4.36-4.27 (5H, m, H-2"', H-3"', H-4"', H-5"', 6"'b-OCH $), 3.84$ (6H, s, OCH3-3', 5'), 3.74 (3H, s, OCH3-3"). ${ }^{13} \mathrm{C}-\mathrm{NMR}$ (pyridine-d5) $\delta 184.1$ (C-4), 165.5 (C-7), 165.4 (C-2), 163.7 (C-5), 159.1 (C-9), 155.3 (C-3', 5'), 149.6 (C-3"), 148.6 (C-4"), 141.9 (C-4'), 135.6 (C-1"), 127.7 (C-1'), 121.8 (C-6"), 117.3 (C-5"), 112.9 (C-2"), 107.8 (C-10), 107.1 (C-3), 106.1 (C-2', 6'), 103.0 (C-1"'), 102.1 (C-6), 96.8 (C-8), 89.4 (C-8"), 80.5 (C-3"'), 79.7 (C-5"'), 76.0 (C-4'"), 75.1 (C-7"), 72.4 (C-2"'), 63.5 (C-6"'), 62.9 (C-9"), 57.7 (C-3', 5'-OCH 3 ), 57.1 (C-3"-OCH $).$

Tricin-4'-O-(erythro- $\beta$-guaiacylglyceryl) ether 7-O- $\beta$-D-glucopyranose (3). Yellow amorphous powder $\left(\mathrm{CH}_{3} \mathrm{OH}\right)$; Negative ESI/MS m/z 687 [M-H] $]^{-}$; IR (KBr) 3364, 2933, 1649, 1607, 1590, 1495, 1457, 1358, 1121, $832 \mathrm{~cm}^{-1}$; ${ }^{1} \mathrm{H}-\mathrm{NMR}$ (pyridine-d5) $\delta 7.57$ (1H, s, H-2"), 7.46 (1H, dd, $\left.J=8.0,1.2 \mathrm{~Hz}, \mathrm{H}-6 "\right)$, 7.29 (2H, s, H-2', 6'), 7.26 (1H, d, $J=8.0 \mathrm{~Hz}, \mathrm{H}-5 "), 7.13$ (1H, d, $J=2.0 \mathrm{~Hz}, \mathrm{H}-8), 6.99$ (1H, s, H-3), $6.86(1 \mathrm{H}, \mathrm{d}, J=2.0 \mathrm{~Hz}, \mathrm{H}-6), 5.77(1 \mathrm{H}, \mathrm{d}, J=6.0 \mathrm{~Hz}, \mathrm{H}-7 "), 5.76\left(1 \mathrm{H}, \mathrm{d}, J=7.2 \mathrm{~Hz}, \mathrm{H}-1{ }^{\prime \prime}\right), 4.95(1 \mathrm{H}$, q, H-8"), 4.54 (1H, br. d, $J=10.4$ Hz, H-6"'a), 4.43 (1H, dd, $J=11.6,4.0$ Hz, H-9"a), 4.37-4.10 (5H, m, H-2"', H-3"', H-4'", H-5"', 6"'b-OCH $), 4.06$ (1H, dd, J = 12.0, 4.0 Hz, H-9"b), 3.82 (6H, s, $\left.\mathrm{OCH}_{3}-3^{\prime}, 5^{\prime}\right), 3.77$ (3H, s, OCH3-3"). ${ }^{13} \mathrm{C}-\mathrm{NMR}$ (pyridine-d5) $\delta 183.5$ (C-4), 165.0 (C-7), 164.9 (C-2), 163.3 (C-5), 158.6 (C-9), 154.6 (C-3', 5'), 149.1 (C-3"), 148.2 (C-4"), 141.9 (C-4'), 134.6 (C-1"), 127.2 (C-1'), 121.4 (C-6"), 116.8 (C-5"), 112.6 (C-2"), 107.4 (C-3), 106.6 (C-10), 105.9 (C-2', 6'), 105.7 (C-1"'), 101.3 (C-6), 96.3 (C-8), 87.8 (C-8"), 80.0 (C-3'"), 79.2 (C-5'"), 75.5 (C-4'"), 74.4 (C-7"), 71.9 (C-2'"), 63.9 (C-6"'), 62.5 (C-9"), $57.6(\mathrm{C}-3$ ', 5'-OCH3), $57.1(\mathrm{C}-3$ "-OCH3).

Tricin-4'-O-(threo- $\beta$-guaiacylglyceryl) ether 7"-O- $\beta$-D-glucopyranose (4). Yellow amorphous powder $(\mathrm{CH} 3 \mathrm{OH})$; HRESI/MS $m / z$ 687.1962 [M-H] $]^{-}$; IR (KBr) 3406, 1639, 1614, 1495, 1358, $1121 \mathrm{~cm}^{-1}$; ${ }^{1} \mathrm{H}-\mathrm{NMR}$ (pyridine- $d 5$ ) and ${ }^{13} \mathrm{C}-\mathrm{NMR}$ (pyridine- $d 5$ ), see Table 1.

Tricin-4'-O-(erythro- $\beta$-guaiacylglyceryl) ether 7"-O- $\beta$-D-glucopyranose (5). Yellow amorphous powder $\left(\mathrm{CH}_{3} \mathrm{OH}\right)$; HRESI/MS $m / z 687.1952[\mathrm{M}-\mathrm{H}]^{-}$; IR (KBr) 3432, 2502, 1685, 1510, 1420, 1399, $1112 \mathrm{~cm}^{-1} ;{ }^{1} \mathrm{H}-\mathrm{NMR}$ (pyridine- $d_{5}$ ) and ${ }^{13} \mathrm{C}-\mathrm{NMR}$ (pyridine- $d_{5}$ ), see Table 1.

Table 1. ${ }^{1} \mathrm{H}-(400 \mathrm{MHz})$ and ${ }^{13} \mathrm{C}-\mathrm{NMR}(100 \mathrm{MHz})$ data of compound 4-5 (in pyridine- $d 5$, $\delta$ in ppm, $J$ in $\mathrm{Hz})$.

\begin{tabular}{ccccc}
\hline \multirow{2}{*}{ No. } & \multicolumn{3}{c}{ Compound 4 } & \multicolumn{2}{c}{ Compound 5 } \\
\cline { 2 - 5 } & $\boldsymbol{\delta}_{\mathbf{C}}$ & $\boldsymbol{\delta}_{\mathbf{H}}$, Coupling Pattern, $\boldsymbol{J}$ in $\mathbf{H z}$ & $\boldsymbol{\delta}_{\mathbf{C}}$ & $\boldsymbol{\delta}_{\mathbf{H}}$, Coupling Pattern, $\boldsymbol{J}$ in $\mathbf{H z}$ \\
\hline 2 & 165.0 & & 165.2 & \\
3 & 107.0 & $6.97, \mathrm{~s}$ & 107.2 & $7.00, \mathrm{~s}$ \\
4 & 183.9 & & 183.9 & \\
5 & 164.3 & & 164.4 & \multirow{2}{*}{$6.74, \mathrm{~d}, 2.0$} \\
6 & 101.3 & $6.72, \mathrm{~d}, 2.0$ & 101.4 & \\
7 & 167.3 & & 167.3 & $6.86, \mathrm{~d}, 2.0$ \\
8 & 96.3 & $6.83, \mathrm{~d}, 2.0$ & 96.3 & \\
9 & 159.7 & & 159.8 & \\
10 & 106.3 & & 106.3 & \\
\hline
\end{tabular}


Table 1. Cont.

\begin{tabular}{|c|c|c|c|c|}
\hline \multirow{2}{*}{ No. } & \multicolumn{2}{|r|}{ Compound 4} & \multicolumn{2}{|r|}{ Compound 5} \\
\hline & $\delta_{\mathrm{C}}$ & $\delta_{\mathrm{H}}$, Coupling Pattern, $\boldsymbol{J}$ in $\mathbf{H z}$ & $\delta_{C}$ & $\delta_{\mathrm{H}}$, Coupling Pattern, $J$ in $\mathrm{Hz}$ \\
\hline $1^{\prime}$ & 127.9 & & 128.2 & \\
\hline $2^{\prime}$ & 105.9 & 7.27 , br. s & 106.0 & 7.29, br. s \\
\hline $3^{\prime}$ & 155.1 & & 155.1 & \\
\hline $4^{\prime}$ & 141.3 & & 142.0 & \\
\hline $5^{\prime}$ & 155.1 & & 155.1 & \\
\hline $6^{\prime}$ & 105.9 & 7.27 , br. s & 106.0 & 7.29, br. s \\
\hline $1^{\prime \prime}$ & 133.1 & & 132.1 & \\
\hline $2^{\prime \prime}$ & 114.0 & $7.50, \mathrm{~d}, 1.6$ & 113.9 & $7.59, \mathrm{~d}, 1.6$ \\
\hline $3^{\prime \prime}$ & 149.4 & & 149.4 & \\
\hline $4 "$ & 148.8 & & 148.9 & \\
\hline $5^{\prime \prime}$ & 117.0 & $7.18, \mathrm{~d}, 8.0$ & 117.1 & $7.21, \mathrm{~d}, 8.0$ \\
\hline $6^{\prime \prime}$ & 122.8 & $7.37, \mathrm{dd}, 8.0,1.6$ & 122.5 & $7.43, \mathrm{dd}, 8.0,1.6$ \\
\hline $7^{\prime \prime}$ & 82.6 & $5.89, \mathrm{~d}, 6.0$ & 81.9 & $6.02, \mathrm{~d}, 4.8$ \\
\hline $8^{\prime \prime}$ & 87.8 & $5.19, \mathrm{~m}$ & 87.6 & $5.16, \mathrm{~m}$ \\
\hline $9 " \mathrm{a}$ & 62.7 & $4.75, \mathrm{dd}, 12.4,3.6$ & 63.0 & $4.44, \mathrm{dd}, 12.0,4.4$ \\
\hline 9"b & & $4.36, \mathrm{dd}, 12.0,2.8$ & & $4.02, \mathrm{dd}, 11.6,5.2$ \\
\hline 3'- $-\mathrm{OCH}_{3}$ & 57.2 & $3.81, \mathrm{~s}$ & 57.7 & $3.83, \mathrm{~s}$ \\
\hline $5^{\prime}-\mathrm{OCH}_{3}$ & 57.2 & $3.81, \mathrm{~s}$ & 57.7 & $3.83, \mathrm{~s}$ \\
\hline 3"- $-\mathrm{OCH}_{3}$ & 56.6 & $3.73, \mathrm{~s}$ & 57.1 & $3.67, \mathrm{~s}$ \\
\hline $1^{\prime \prime \prime}$ & 105.7 & $5.54, \mathrm{~d}, 8.0$ & 105.6 & $5.35, \mathrm{~d}, 8.0$ \\
\hline $2^{\prime \prime \prime}$ & 75.5 & & 77.1 & \\
\hline $3^{\prime \prime \prime}$ & 79.7 & $420412 \mathrm{~m}$ & 80.1 & 421200 \\
\hline $4^{\prime \prime \prime}$ & 71.9 & $4.28-4.12, \mathrm{~m}$ & 72.8 & $4.31-3.09, \mathrm{~m}$ \\
\hline $5^{\prime \prime \prime}$ & 79.6 & & 79.7 & \\
\hline 6"'a & 63.0 & $4.41, \mathrm{~m}$ & 63.8 & $4.45, \mathrm{~m}$ \\
\hline 6"'b & & $4.20, \mathrm{~m}$ & & $4.30, \mathrm{~m}$ \\
\hline
\end{tabular}

\section{Conclusions}

Five compounds were isolated from the aerial parts of Zizania latifolia using a prep-LC instrument equipped with cartridges of various sizes and were identified based on spectroscopic data analysis, including NMR, UV, and ESI-MS. Four flavonolignan glycosides, including two new flavonolignan glycosides, showed structural similarities in being derived from tricin-7- $O-\beta$-D-glucopyranose (1), and were shown to be diastereomers of each other. Tricin-4'-O-(threo- $\beta$-guaiacylglyceryl) ether 7-O- $\beta$-Dglucopyranose (2) and tricin-4'-O-(erythro- $\beta$-guaiacylglyceryl) ether 7-O- $\beta$-D-glucopyranose (3) were shown to be diastereomers distinguished by threo and erythro configurations, whereas tricin-4'-O-(threo- $\beta$-guaiacylglyceryl) ether 7"-O- $\beta$-D-glucopyranose (4) and tricin-4'-O-(erythro- $\beta$ guaiacylglyceryl) ether 7"-O- $\beta$-D-glucopyranose (5) were shown to be threo and erythro, respectively.

\section{Supplementary Materials}

${ }^{1} \mathrm{H}-\mathrm{NMR},{ }^{13} \mathrm{C}-\mathrm{NMR}$, and HR-ESIMS spectra of $\mathbf{4 - 5}$ are available as supporting data. Supplementary materials can be accessed at: http://www.mdpi.com/1420-3049/20/04/5616/s1. 


\section{Acknowledgments}

This work was supported by the Next Generation Bio-Green 21(PJ008020) Project from Rural Development Administration, Republic of Korea, and a grant from Kyung Hee University in 2009 (KHU-20100160), and by a grant from the regional innovation center program of the Ministry of Trade, Industry and Energy at the Skin Biotechnology Center of Kyung Hee University, Korea.

\section{Author Contributions}

M.-H.B., N.-I.B., and D.-K.C. designed research; S.-S.L., Y.-S.B., and M.-C.S. performed research and analyzed the data; S.-S.L. wrote the paper. All authors read and approved the final manuscript.

\section{Conflicts of Interest}

The authors declare no conflict of interest.

\section{References}

1. Oelke, E.A.; Porter, R.A.; Gramcher, A.W.; Addis, P.B. Wild rice-new interest in old crop. Cereal Foods World 1997, 42, 234-247.

2. Guo, H.B.; Li, S.M.; Peng, J.; Ke, W.D. Zizania latifolia Trucz. cultivated in China. Genet. Resour. Crop Evol. 2007, 54, 1211-1217.

3. Han, S.F.; Zhang, H.; Zhai, C.K. Protective potentials of wild rice (Zizania latifolia (Griseb) Turcz) against obesity and lipotoxicity induced by a high-fat/cholesterol diet in rats. Food Chem. Toxicol. 2012, 50, 2236-2269.

4. Zhang, H.; Cao, P.; Agellon, L.B.; Zhai, C.K. Wild rice (Zizania latifolia (Griseb) Turcz) improves the serum lipid profile and antioxidant status of rats fed with a high fat/cholesterol diet. Br. J. Nutr. 2009, 102, 1723-1727.

5. Lee, S.S.; Baek, Y.S.; Eun, C.S.; Yu, M.H.; Baek, N.I.; Chung, D.K.; Bang, M.H.; Yang, S.A. Tricin derivatives as anti-inflammatory and anti-allergic constituents from the aerial part of Zizania latifolia. Biosci. Biotechnol. Biochem. 2015, 6, 1-7.

6. Bouaziz, M.; Veitch, N.C.; Grayer, R.J.; Simmonds, M.S.J.; Damak, M. Flavolignans from Hyparrhenia hirta. Phytochemistry 2002, 60, 515-520.

7. Jiao, J.; Zang, Y.; Liu, C.; Liu, J.; Wu, X.; Zang, Y. Separation and purification of tricin from an antioxidant product derived from bamboo leaves. J. Agric. Food Chem. 2007, 55, 10086-10092.

8. Jeong, R.H.; Lee, D.Y.; Cho, J.G.; Lee, S.M.; Kang, H.C.; Seo, W.D.; Kang, H.W.; Kim, J.Y.; Baek, N.I. A new flavonolignan from the arial parts of Oryza sativa L. inhibits nitric oxide production in RAW 264.7 macrophage cells. J. Korean Soc. Appl. Biol. Chem. 2011, 54, 865-870.

9. Nakajima, Y.; Yun, Y.S.; Kunugi, A. Six new flavonolignans from Sasa veitchii (Carr.) Rehder. Tetrahedron 2003, 59, 8011-8015.

10. Jung, Y.J.; Park, J.H.; Cho, J.G.; Seo, K.H.; Lee, D.S.; Kim, Y.C.; Kang, H.C.; Song, M.C.; Baek, N.I. Lignan and flavonoids from the stems of Zea mays and their anti-inflammatory and nueroprotective activities. Arch. Pharm. Res. 2014, 37, 1978-2014. 
11. Jeong, R.H.; Lee, D.Y.; Cho, J.G.; Seo, K.H.; Lee, J.W.; Lee, M.H.; Seo, W.D.; Kang, H.C.; Kim, G.S.; Noh, H.J.; et al. New flavonolignan glucoside from the aerial parts of Oryza Sativa. Chem. Nat. Compd. 2013, 49, 1003-1005.

Sample Availability: Samples of the compounds 1-5 are available from the authors.

(C) 2015 by the authors; licensee MDPI, Basel, Switzerland. This article is an open access article distributed under the terms and conditions of the Creative Commons Attribution license (http://creativecommons.org/licenses/by/4.0/). 